\title{
Digital Multichannel Photometer
}

A new digital multichannel photometer system has been developed for use in astronomy and other research areas concerned with the detection of faint-light images. The photometer system is comparable in performance to a good photomultiplier tube array except that digital electronics are used instead of analog. The digital circuits allow substantial reductions in cost, weight, and size of the system.

The photometer system incorporates a conventional, single-stage, magnetically-focused diode tube as shown in Figure 1. Light coming from a faint image is converted into electron current at the cesium anti-

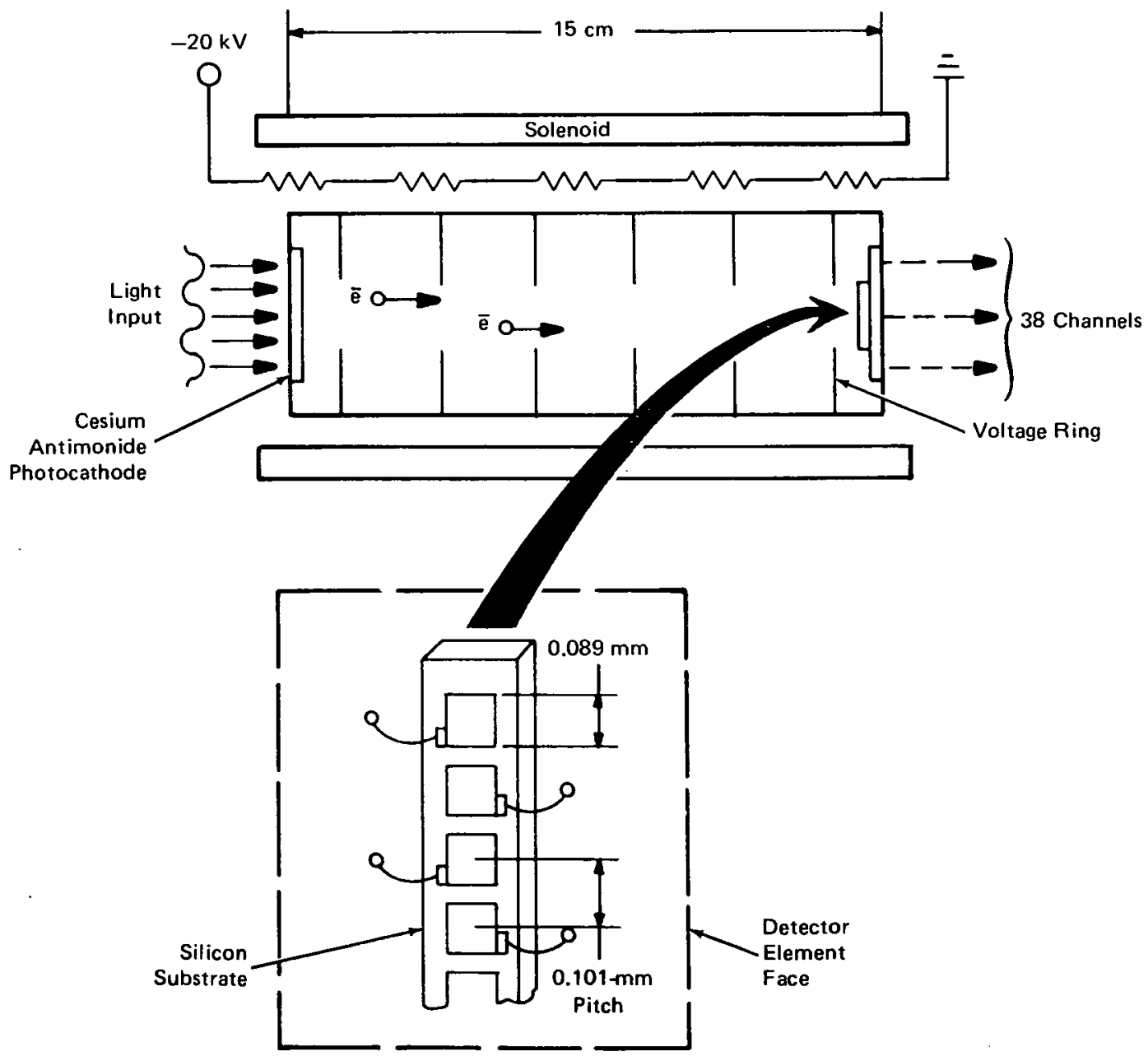

Figure 1. Diode Tube 


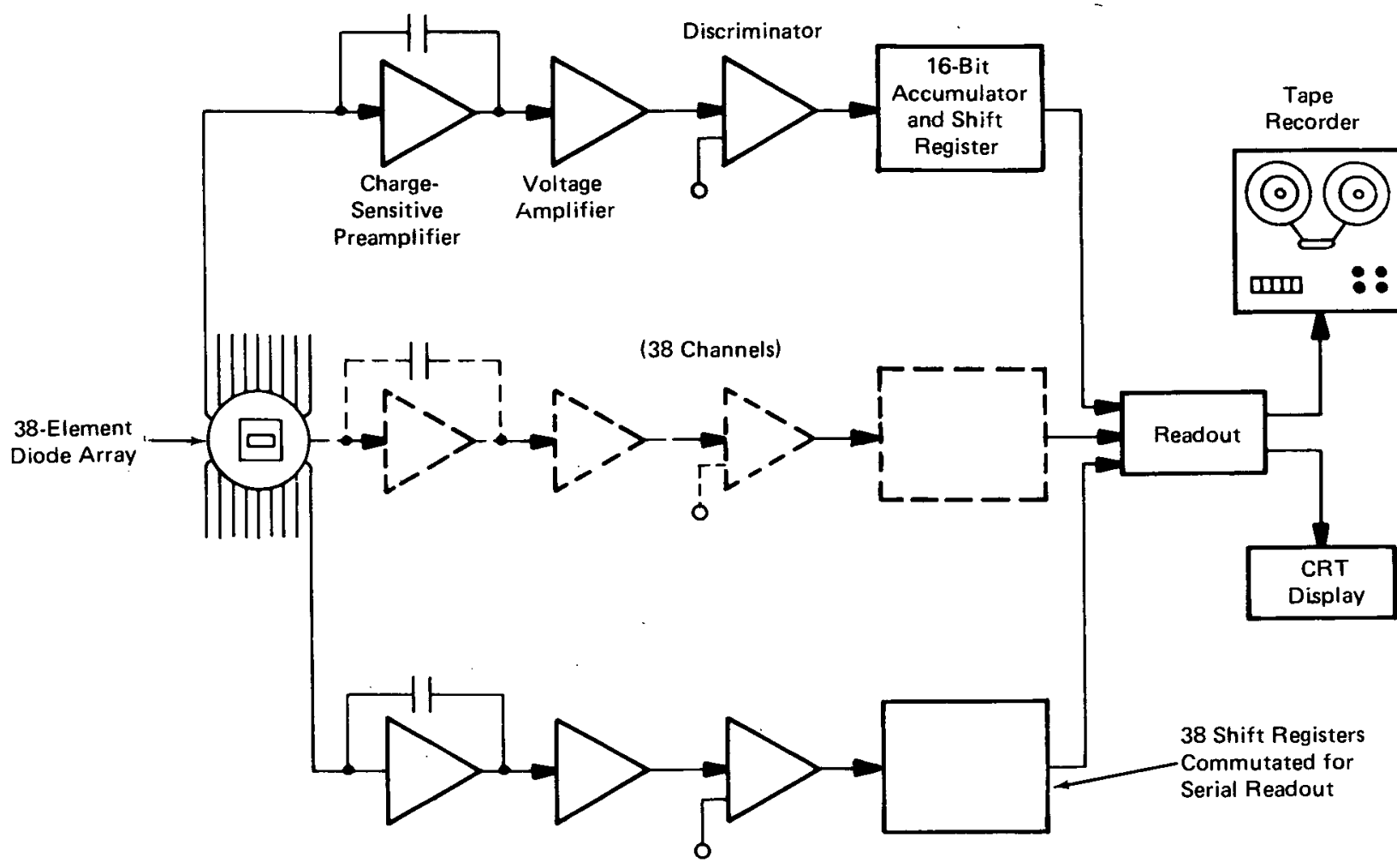

Figure 2. Photometer System

monide photocathode. These electrons are accelerated through a $20-\mathrm{kV}$ potential, reaching energies of $20 \mathrm{keV}$. They are focused onto a linear diode array comprising 38 diffused $p$-n diodes on a silicon wafer.

Each diode is 0.089 - by $0.089-\mathrm{mm}$ square connected to a separate circuit channel (see Figure 2). Every channel includes a charge-sensitive preamplifier, an amplifier, a discriminator, and a digital accumulator for the detection of photoelectron arrivals. As each electron enters the diode depletion region, an electronhole pair is produced for every $3.5-\mathrm{eV}$ energy loss. Thus, an electron losing $16 \mathrm{keV}$ of energy in the depletion region produces a charge pulse of approximately $7.3 \times 10^{-16}$ coulombs. This charge is easily detected by the charge-sensitive preamplifier.

The preamplifiers are constructed using low-noise field-effect transistors. However, there is a low-energy noise component in the preamplifier output which is eliminated in the discriminator. The output is fed to a 16-bit accumulator and shift register which accumulates the image information and feeds it serially to a tape recorder or a cathode-ray tube (CRT) display, while the next accumulation is taking place.

Results show that the tube dark current at $20 \mathrm{kV}$ and a temperature of $35^{\circ} \mathrm{C}$ is approximately 200 electrons $/ \mathrm{s} / \mathrm{cm}^{2}$, which suggests that most of the dark current is due to thermionic emission of the photo- cathode. Since each detector is approximately $10^{-4}$ $\mathrm{cm}^{2}$ in area, the dark-current rate per diode is approximately 0.02 count $/ \mathrm{s}$. The present tube design can be expanded to include 1000 diode elements. In addition, different diode arrays can be generated for different applications.

Note:

Requests for further information may be directed to:

Technology Utilization Officer

NASA Headquarters

Code KT

Washington, D. C. 20546

Reference: TSP74-10200

\section{Patent status:}

Title to this invention has been waived under the provisions of the National Aeronautics and Space Act [42 U.S.C. 2457(f)], to the University of California, Berkeley, California 94720.

Source: Edward A. Beaver and Carl E. McIlwain of University of California under contract to NASA Headquarters (HQN-10791)

Category 03, 02 